Research Article

\title{
Heat and Mass Transfer of Rotational Flow of Unsteady Third-Grade Fluid over a Rotating Cone with Buoyancy Effects
}

\author{
S. Saleem \\ Department of Mathematics, College of Science, King Khalid University, Abha 61413, Saudi Arabia \\ Correspondence should be addressed to S. Saleem; saakhtar@kku.edu.sa
}

Received 18 February 2021; Revised 18 March 2021; Accepted 25 March 2021; Published 8 April 2021

Academic Editor: Praveen Agarwal

Copyright (c) 2021 S. Saleem. This is an open access article distributed under the Creative Commons Attribution License, which permits unrestricted use, distribution, and reproduction in any medium, provided the original work is properly cited.

\begin{abstract}
Objective of this paper is a study of the impact of heat and mass transfer on time-dependent flow of a third-grade convective fluid due to an infinitely rotating upright cone. An interesting fact is observed that the similarity solutions only exist, if we take the angular velocities of the cone and far away from the fluid as an inverse function of time. The analytical solutions of the reduced ordinary differential equations of third-grade fluids are offered by the optimal homotopy analysis method (OHAM). The results for important parameters are illustrated graphically as well as in tabular form. The precision of the present results is also checked by comparison with the numerical outcomes published earlier. The impact of non-Newtonian fluid parameters is found to decrease the primary skin-friction coefficient. There is an aggregate in Nusselt and Sherwood numbers for increasing the ratio of the buoyancy forces.
\end{abstract}

\section{Introduction}

In the modern era, non-Newtonian fluid flows gain appreciable attention due to its huge applications in massive engineering and industrial processes. This phenomenon achieves great importance in theoretical as well as practical viewpoint. Heat and mass transfer in non-Newtonian fluids are of significance in various manufacturing, industrial, and engineering applications such as food processing, nanomaterial production, and lubrication.

Nazir et al. [1] studied transport phenomenon in Carreau fluid with variable diffusion. Makinde and Chinyoka [2] reported numerical study of unsteady hydromagnetic generalized Couette flow of a reactive third-grade fluid with asymmetric convective cooling. Generalized Couette flow of a third-grade fluid with slip: the exact solutions were analyzed by Ellahi et al. [3]. Salahuddin et al. [4] found the solutions of MHD flow of Williamson fluid over a stretching sheet. Attia et al. [5] explored unsteady Couette flow of a thermally conducting viscoelastic fluid with porosity. Krishna et al. [6] offered multiple solutions for non-Newtonian fluids along an elongating surface. Hayat et al. [7] discussed impressions of radiations and chemical reactions in stretched flows of Jeffrey fluids. Properties of hall and ion slip with Jeffrey peristaltic fluid were deliberated by Ellahi et al. [8]. The process of mixed convection exists when induced and normal convection variations are compatible. It has a marvelous role heat exchangers, solar collectors and nuclear reactors. In the current work, a vertically aligned cone is fixed in a non-Newtonian fluid with the outward flow is inspected. Problems related to heat transfer with convective flow about conic shaped objects are superbly applied in automobile and organic productions. In numerous realworld situations, the unsteady combined convective flows do not essentially give similarity solution and for the past era, several problems have been examined, where the nonsimilarity has to be measured. The body arc is responsible for fluid flow for free stream velocity. The essential scientific complications intricate in finding nonsimilar solutions for such studies have limited many scientific minds to confine their findings either to the nonsimilar steady flows or to the unsteady semisimilar flows. In self-similar solutions, a set of partial differential equations can be converted to a scheme of ordinary differential equations. In modern studies, Anilkumar and Roy [9] and Saleem et al. [10] did an important work for mixed convection flow on a rotating cone in a 
rotating fluid. Significant features of Dufour and Soret effect on viscous fluid by a rotating cone with entropy generation were inspected by Khan et al. [11]. The variable surface temperature and heat flow on a vertical permeable circular cone was inspected on a free convection by Hassainetal et al. [12]. Shamkha et al. [13] examined the analysis of radiation impacts on a cone embedded in a porous nanofluid-filled medium in a mixed convection. There are numerous developments in flows depending on time and with surface mass transfer (suction/injection), where the thermal and mass diffusion of temperature and concentration gradient results in the buoyancy effects. Therefore, it is worthwhile studying the boundary layer fluid over a cone with the thermal diffusion and mass diffusion if the freely flow rate changes randomly with time [14-18] in order to improve the examination on mutual convection.

The main task of the existing paper is to observe the variation of heat and mass transfer on third-grade fluid flow induced by the rotating cone. The highly nonlinear ordinary differential equations of the third-grade fluid with the prescribed wall temperature conditions are solved by the optimal homotopy analysis method (OHAM) [19-27]. Usually, the nonlinear system of ODE is difficult to tackle with the analytical method. Mostly, some well-known techniques such as perturbation methods are applied for this purpose. These techniques have limitations as they rely on small/large parameters. Moreover, such algorithms fail to control the convergence region of series solutions. Liao [19] was the first who developed a semianalytical method to handle highly nonlinear equations with the specialty to regulate and control the region of convergence.

Furthermore, graphs and numerical tables show the effects of interesting parameters on the velocities, stress tensor, temperature, and concentration fields. In addition, comparisons of the findings with the literature previously available have verified the exactness of the analytical methodology.

\section{Mathematical Model}

Let consider the laminar incompressible flow of a thirdgrade fluid on a rotational vertical cone in a rotating fluid. The motion is unsteady due to the rotation of the cone and the fluid along the cone axis. The geometry of flow field is described in Figure 1.

A rectangular coordinate system is considered where $u, v$, and $w$ are the velocity components in the $x$-tangential, $y$-azimuthal, and $z$-normal directions, respectively. Axisymmetric flow is considered while wall temperature $T_{w}$ and the wall concentration $C_{w}$ vary linearly in $x$. The momentum, energy, and diffusion equations for a third-grade fluid are given as [9]

$$
\begin{aligned}
\frac{\partial(x u)}{\partial x}+\frac{\partial(x w)}{\partial z}= & 0 \\
\frac{\partial u}{\partial t}+u \frac{\partial u}{\partial x}+w \frac{\partial u}{\partial z}-\frac{v^{2}}{x}= & -\frac{v_{e}^{2}}{x}+v \frac{\partial^{2} u}{\partial z^{2}}+\frac{\alpha_{1}}{\rho}\left\{\frac{\partial^{3} u}{\partial z^{2} \partial t}+\frac{\partial u}{\partial x} \frac{\partial^{2} u}{\partial z^{2}}+\frac{\partial^{2} v}{\partial x} \frac{\partial v}{\partial z}+\frac{\partial v}{\partial x} \frac{\partial^{2} v}{\partial z^{2}}\right\} \\
& +\frac{\alpha_{2}}{\rho}\left\{\frac{1}{x}\left(\frac{\partial u}{\partial z}\right)^{2}+\frac{\partial^{2} u}{\partial x} \frac{\partial u}{\partial z}-\frac{1}{x}\left(\frac{\partial v}{\partial z}\right)^{2}+\frac{\partial u}{\partial z} \frac{\partial^{2} w}{\partial z^{2}}+\frac{\partial w}{\partial z} \frac{\partial^{2} u}{\partial z^{2}}\right\}+\frac{\left(\alpha_{1}+\alpha_{2}\right)}{\rho} \\
& \left\{3 \frac{\partial^{2} u}{\partial x} \frac{\partial u}{\partial z}+2 \frac{\partial u}{\partial x} \frac{\partial^{2} u}{\partial z^{2}}+\frac{\partial^{2} v}{\partial x} \frac{\partial v}{\partial z}+\frac{\partial v}{\partial x} \frac{\partial^{2} v}{\partial z^{2}}-\frac{v}{x} \frac{\partial^{2} v}{\partial z^{2}}-\frac{1}{x}\left(\frac{\partial v}{\partial z}\right)^{2}+\frac{\partial u}{\partial z} \frac{\partial^{2} w}{\partial z^{2}}+\frac{\partial w}{\partial z} \frac{\partial^{2} u}{\partial z^{2}}\right\} \\
& +\frac{\beta_{3}}{\rho}\left[2 \frac{\partial^{2} u}{\partial z^{2}}\left\{3\left(\frac{\partial u}{\partial z}\right)^{2}+\left(\frac{\partial v}{\partial z}\right)^{2}+4 \frac{\partial u}{\partial z} \frac{\partial v}{\partial z} \frac{\partial^{2} v}{\partial z^{2}}\right\}\right]+g \xi \cos \alpha^{*}\left(T-T_{\infty}\right)+g \xi^{*} \cos \alpha^{*}\left(C-C_{\infty}\right),
\end{aligned}
$$

$$
\begin{aligned}
\frac{\partial v v}{\partial t}+u \frac{\partial v}{\partial x}+w \frac{\partial v}{\partial z}-\frac{u v}{x}= & -\frac{\partial v_{e}}{\partial t}+u \frac{\partial^{2} v}{\partial z^{2}}+\frac{\alpha_{1}}{\rho}\left\{\frac{\partial^{3} v}{\partial z^{2} \partial t}-\frac{v}{x} \frac{\partial^{2} u}{\partial z^{2}}+\frac{u}{x} \frac{\partial^{2} v}{x} \frac{\partial z^{2}}{\partial}\right\} \\
& +\frac{\alpha_{2}}{\rho}\left\{\frac{\partial u}{\partial z} \frac{\partial^{2} v}{\partial x}+\frac{\partial v}{\partial z} \frac{\partial^{2} u}{\partial x}+\frac{\partial v}{\partial z} \frac{\partial^{2} w}{\partial z^{2}}+\frac{\partial w}{\partial z} \frac{\partial^{2} v}{\partial z^{2}}\right\}+\frac{\left(\alpha_{1}+\alpha_{2}\right)}{\rho} \\
& \left\{\frac{\partial^{2} v}{\partial x \partial z} \frac{\partial u}{\partial z}+\frac{\partial v}{\partial x} \frac{\partial^{2} u}{\partial z^{2}}-\frac{v}{x} \frac{\partial^{2} v}{\partial z^{2}}+\frac{2 u}{x} \frac{\partial^{2} v}{\partial z^{2}}+\frac{1}{x} \frac{\partial u}{\partial z} \frac{\partial v}{\partial z}+\frac{\partial v}{\partial z} \frac{\partial^{2} w}{\partial z^{2}}+\frac{\partial w}{\partial z} \frac{\partial^{2} v}{\partial z^{2}}\right\} \\
& +\frac{\beta_{3}}{\rho}\left[2 \frac{\partial^{2} v}{\partial z^{2}}\left\{\left(\frac{\partial u}{\partial z}\right)^{2}+3\left(\frac{\partial v}{\partial z}\right)^{2}+4 \frac{\partial u}{\partial z} \frac{\partial v}{\partial z} \frac{\partial^{2} u}{\partial z^{2}}\right\}\right], \\
& \frac{\partial T}{\partial t}+u \frac{\partial T}{\partial x}+w \frac{\partial T}{\partial z}=\kappa \frac{\partial^{2} T}{\partial z^{2}},
\end{aligned}
$$




$$
\frac{\partial C}{\partial t}+u \frac{\partial C}{\partial x}+w \frac{\partial C}{\partial z}=D_{B} \frac{\partial^{2} C}{\partial z^{2}}
$$

The appropriate physical boundary conditions are defined as

$$
\begin{aligned}
u(x, 0, t) & =w(x, 0, t)=0, \\
v(x, 0, t) & =\Omega_{1} x \operatorname{Sin} \alpha^{*}\left(1-s t^{*}\right)^{-1}, \\
k T_{z}(x, 0, t) & =-q_{w}, \rho D C_{z}(x, 0, t)=-m_{w}, \\
u(x, \infty, t) & =v(x, \infty, t)=\Omega_{2} x \operatorname{Sin} \alpha^{*}\left(1-s t^{*}\right)^{-1}, \\
T(x, \infty, t) & =T_{\infty}, C(x, \infty, t)=C_{\infty},
\end{aligned}
$$

where $\alpha_{i}(i=1,2)$ and $\beta_{i}(i=1,2,3)$ are the material parameters of fluid considered. Note that the viscous dissipation effects in the energy equation are assumed to be negligible. Introducing the following nondimensional quantities [9],

$$
\begin{aligned}
v_{e} & =\Omega_{2} x \sin \alpha^{*}\left(1-s t^{*}\right)^{-1}, \eta=\left(\frac{\Omega \sin \alpha^{*}}{v}\right)^{1 / 2}\left(1-s t^{*}\right)^{-1 / 2} z, \gamma=\frac{\Omega_{1}}{\Omega}, \\
t^{*} & =\left(\Omega \sin \alpha^{*}\right) t, u(t, x, z)=-2^{-1} \Omega x \sin \alpha^{*}\left(1-s t^{*}\right)^{-1} f^{\prime}(\eta), \\
v(t, x, z) & =\Omega x \sin \alpha^{*}\left(1-s t^{*}\right)^{-1} g(\eta), w(t, x, z)=\left(v \Omega \sin \alpha^{*}\right)^{1 / 2}\left(1-s t^{*}\right)^{-1 / 2} f(\eta), \\
T(t, x, z)-T_{\infty} & =\left(T_{w}-T_{\infty}\right) \theta(\eta),\left(T_{w}-T_{\infty}\right)=\left(T_{0}-T_{\infty}\right) \frac{x}{L}\left(1-s t^{*}\right)^{-2}, \\
C(t, x, z)-C_{\infty} & =\left(C_{w}-C_{\infty}\right) \phi(\eta),\left(C_{w}-C_{\infty}\right)=\left(C_{0}-C_{\infty}\right) \frac{x}{L}\left(1-s t^{*}\right)^{-2}, \\
\left(T_{0}-T_{\infty}\right) \frac{L^{3}}{v^{2}}, \operatorname{Re}_{L} & =\Omega \sin \alpha^{*} \frac{L^{2}}{v}, \lambda_{1}=\frac{G r_{1}}{\operatorname{Re}_{L}^{2}}, \\
\left(C_{0}-C_{\infty}\right) \frac{L^{3}}{v^{2}}, \lambda_{2} & =\frac{G r_{2}}{\operatorname{Re}_{L}^{2}}, N=\frac{\lambda_{2}}{\lambda_{1}}, \operatorname{Pr}=\frac{v}{\kappa}, S c=\frac{v}{D_{B}}, \\
\varepsilon_{1} & =\frac{\alpha_{1} \Omega \sin \alpha^{*}\left(1-s t^{*}\right)^{-1}}{\mu}, \varepsilon_{2}=\frac{\alpha_{2} \Omega \sin \alpha^{*}\left(1-s t^{*}\right)^{-1}}{\mu}, \varepsilon_{3}=\frac{\beta_{3}\left(\Omega \sin \alpha^{*}\right)^{3}\left(1-s t^{*}\right)^{-3}}{v \mu},
\end{aligned}
$$

$G r_{1=} g \xi \cos \alpha^{*}\left(T_{0}-T_{\infty}\right) \frac{L^{3}}{v^{2}}, \operatorname{Re}_{L}=\Omega \sin \alpha^{*} \frac{L^{2}}{v}, \lambda_{1}=\frac{G r_{1}}{\operatorname{Re}_{L}^{2}}$,

$G r_{2=} g \xi^{*} \cos \alpha^{*}\left(C_{0}-C_{\infty}\right) \frac{L^{3}}{v^{2}}, \lambda_{2}=\frac{G r_{2}}{\operatorname{Re}_{L}^{2}}, N=\frac{\lambda_{2}}{\lambda_{1}}, \operatorname{Pr}=\frac{v}{\kappa}, S c=\frac{v}{D_{B}}$,

where flow field accelerates for positive $s$ (unsteady parameter) and vice versa. $\lambda_{1}$ is the buoyancy force parameter, $N$ is the ratio of the buoyancy forces, and $\varepsilon_{i}(i=1,2,3)$ are the third-grade fluid parameters. The governing equation along with boundary conditions (1-6) is systematically satisfied and written as

$$
\begin{gathered}
\left(1+\varepsilon_{1} s\right) f^{\prime \prime \prime}-\left(f+\frac{1}{2} s \eta\right) f^{\prime \prime}+\left(\frac{1}{2} f^{\prime}-s\right) f^{\prime}-2\left\{g^{2}-(1-\gamma)^{2}\right\}-2 \lambda_{1}(\theta+N \phi) \\
-\left(\varepsilon_{1}+\varepsilon_{2}-\frac{1}{4} \varepsilon_{3} f^{\prime \prime \prime}\right) f^{\prime \prime 2}-\left(\varepsilon_{1}-2 \varepsilon_{2}\right) f^{\prime \prime} f^{\prime \prime \prime}-2\left(\varepsilon_{1}+\varepsilon_{2}-\frac{1}{4} \varepsilon_{3} f^{\prime \prime \prime}\right) g^{\prime \prime 2}-2\left(\varepsilon_{1}-\frac{1}{4} \varepsilon_{3} f^{\prime \prime}\right) g g^{\prime \prime}=0 \\
\left(1-\varepsilon_{1}\right) g^{\prime \prime}-\left(f g^{\prime}-g f^{\prime}\right)+s\left(1-\gamma-g-\frac{1}{2} \eta g^{\prime}\right)-\frac{1}{2} \varepsilon_{1} s \eta g^{\prime \prime \prime}+\left(\frac{3}{2} \varepsilon_{1}+\varepsilon_{2}\right) f^{\prime \prime \prime} g-\left(\frac{1}{2} \varepsilon_{1}+\varepsilon_{2}\right) f^{\prime} g^{\prime \prime}-\varepsilon_{3}\left\{\left(f^{\prime \prime}\right)^{2}+6\left(g^{\prime}\right)^{2}\right\} g^{\prime \prime}=0
\end{gathered}
$$

$$
\frac{1}{\operatorname{Pr}} \theta^{\prime \prime}-\left(f \theta^{\prime}-f^{\prime} \frac{\theta}{2}\right)-s\left(2 \theta+2^{-1} \eta \theta^{\prime}\right)=0
$$




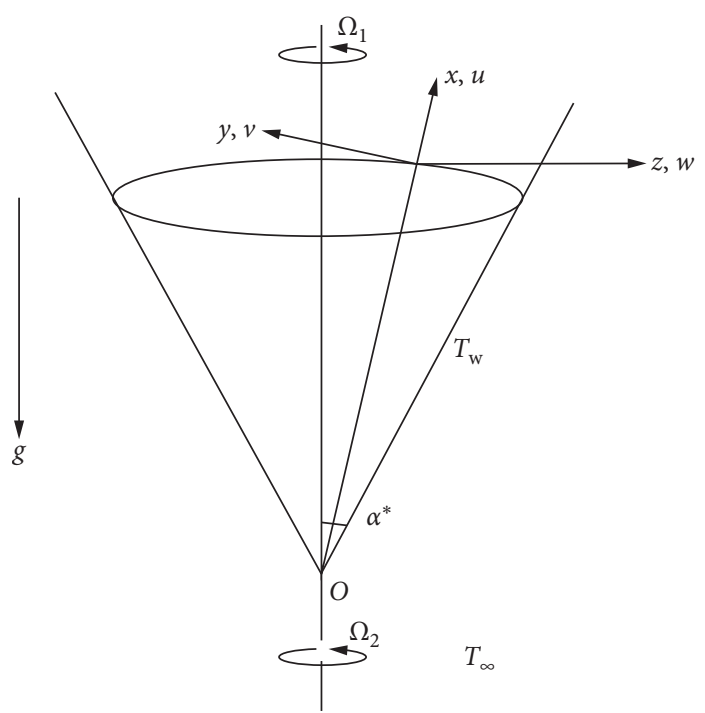

Figure 1: Geometry of the flow field.

$$
\begin{gathered}
\frac{1}{S c} \phi^{\prime \prime}-\left(f \phi^{\prime}-f^{\prime} \frac{\phi}{2}\right)-s\left(2 \phi+2^{-1} \eta \phi^{\prime}\right)=0, \\
f(0)=0=f^{\prime}(0), g(0)=\gamma, \theta(0)=\phi(0)=1, \\
f^{\prime}(\infty)=0=f^{\prime \prime}(\infty), g(\infty)=1-\gamma, g^{\prime}(\infty)=0, \theta(\infty)=\phi(\infty)=0 .
\end{gathered}
$$

The skin-friction coefficients in the primary and sec- where ondary directions are, respectively, given by

$$
\begin{aligned}
C_{f x} & =\frac{\left[2 \tau_{x z}\right]_{z=0}}{\rho\left[\Omega x \sin \alpha^{*}\left(1-s t^{*}\right)^{-1}\right]^{2}}, \\
C_{f y} & =\frac{\left[2 \tau_{y z}\right]_{z=0}}{\rho\left[\Omega x \sin \alpha^{*}\left(1-s t^{*}\right)^{-1}\right]^{2}},
\end{aligned}
$$

$$
\begin{aligned}
& C_{f x} \operatorname{Re}_{x}^{1 / 2}=\left[-f^{\prime \prime}+\varepsilon_{1}\left(2 g g^{\prime}-\frac{3}{2} s f^{\prime \prime}+\frac{1}{2} f^{\prime} f^{\prime \prime}-s \frac{\eta}{2} f^{\prime}\right)-\varepsilon_{2} f^{\prime} f^{\prime \prime}-6 \varepsilon_{3} f^{\prime 2} f^{\prime \prime}\right]_{\eta=0}, \\
& C_{f y} \operatorname{Re}_{x}^{1 / 2}=\left[-g^{\prime}-\varepsilon_{1}\left(\frac{3}{2} s g^{\prime}-\frac{1}{2} f^{\prime} g^{\prime}+\frac{1}{2} f^{\prime \prime} g+s \frac{\eta}{2} g^{\prime}\right)-\varepsilon_{2} f^{\prime} g^{\prime}-\varepsilon_{3} f^{\prime 2} g^{\prime}\right]_{\eta=0},
\end{aligned}
$$

where $\operatorname{Re}_{x}=\left(x^{2} \Omega \sin \alpha^{*}\left(1-s t^{*}\right)^{-1} / v\right)$.

The rate of heat and mass transfer in nondimensional form is written as

$$
\begin{aligned}
& \operatorname{NuRe}_{x}^{-1 / 2}=-\theta^{\prime}(0), \\
& \operatorname{ShRe}_{x}^{-1 / 2}=-\phi^{\prime}(0) .
\end{aligned}
$$

\section{Solution Procedure}

In order to get the analytical solutions of equations (8) to (12), we have applied the optimal homotopy analysis method (OHAM) [19-27]. The base function for velocity fields $f(\eta), g(\eta)$, temperature field $\theta(\eta)$, and the concentration field $\phi(\eta)$ can be stated as 


$$
\left\{\eta^{k} \exp (-n \eta) \mid k \geq 0 . n \geq 0\right\}
$$

in the form

$$
\begin{aligned}
& f(\eta)=a_{0,0}^{0}+\sum_{n=0}^{\infty} \sum_{k=0}^{\infty} a_{m, n}^{k} \eta^{k} \exp (-n \eta) \\
& g(\eta)=b_{0,0}^{0}+\sum_{n=0}^{\infty} \sum_{k=0}^{\infty} b_{m, n}^{k} \eta^{k} \exp (-n \eta) \\
& \theta(\eta)=\sum_{n=0}^{\infty} \sum_{k=0}^{\infty} c_{m, n}^{k} \eta^{k} \exp (-n \eta) \\
& \phi(\eta)=\sum_{n=0}^{\infty} \sum_{k=0}^{\infty} d_{m, n}^{k} \eta^{k} \exp (-n \eta)
\end{aligned}
$$

in which $a_{m, n}^{k}, b_{m, n}^{k}, c_{m, n}^{k}$, and $d_{m, n}^{k}$ are the coefficients. The initial guesses $f_{0}, g_{0}, \theta_{0}$, and $\phi_{0}$ are

$$
\begin{aligned}
& f_{0}(\eta)=0 \\
& g_{0}(\eta)=(1-\gamma)+(2 \gamma-1) \exp (-\eta) \\
& \theta_{0}(\eta)=\exp (-\eta) \\
& \phi_{0}(\eta)=\exp (-\eta) \\
& \zeta_{f}(\eta)=\frac{\mathrm{d}^{3} f}{\mathrm{~d} \eta^{3}}-\frac{\mathrm{d} f}{\mathrm{~d} \eta} \\
& \zeta_{g}(\eta)=\frac{\mathrm{d}^{2} g}{\mathrm{~d} \eta^{2}}-g \\
& \zeta_{\theta}(\eta)=\frac{d^{2} \theta}{d \eta^{2}}-\theta \\
& \zeta_{\phi}(\eta)=\frac{\mathrm{d}^{2} \phi}{\mathrm{d} \eta^{2}}-\phi
\end{aligned}
$$

3.1. Optimal Convergence-Control Parameters. All series solution of homotopic type involves the nonzero auxiliary parameters $c_{0}^{f}, c_{0}^{g}, c_{0}^{\theta}$, and $c_{0}^{\phi}$ which describe the convergence region. To find out the optimal values of $c_{0}^{f}, c_{0}^{g}, c_{0}^{\theta}$, and $c_{0}^{\phi}$, we have utilized the notion of minimization as introduced by [19]

$$
E_{m}^{f}=\frac{1}{k+1} \sum_{j=0}^{k}\left\{N_{f}\left(\sum_{i=0}^{m} \hat{f}(\eta), \sum_{i=0}^{m} \hat{g}(\eta), \sum_{i=0}^{m} \hat{\theta}(\eta), \sum_{i=0}^{m} \widehat{\phi}(\eta)\right)_{\eta=j \delta \eta}\right\}^{2} \mathrm{~d} \eta,
$$

$$
E_{m}^{g}=\frac{1}{k+1} \sum_{j=0}^{k}\left\{N_{g}\left(\sum_{i=0}^{m} \hat{f}(\eta), \sum_{i=0}^{m} \hat{g}(\eta)\right)_{\eta=j \delta \eta}\right\}^{2} \mathrm{~d} \eta
$$

$$
E_{m}^{\theta}=\frac{1}{k+1} \sum_{j=0}^{k}\left\{N_{\theta}\left(\sum_{i=0}^{m} \widehat{f}(\eta), \sum_{i=0}^{m} \widehat{\theta}(\eta), \sum_{i=0}^{m} \widehat{\phi}(\eta)\right)_{\eta=j \delta \eta}\right\}^{2} \mathrm{~d} \eta
$$

$$
E_{m}^{\phi}=\frac{1}{k+1} \sum_{j=0}^{k}\left\{N_{\phi}\left(\sum_{i=0}^{m} \widehat{f}(\eta), \sum_{i=0}^{m} \widehat{\theta}(\eta), \sum_{i=0}^{m} \widehat{\phi}(\eta)\right)_{\eta=j \delta \eta}\right\}^{2} \mathrm{~d} \eta
$$

$$
E_{m}^{t}=E_{m}^{f}+E_{m}^{g}+E_{m}^{\theta}+E_{m}^{\phi}
$$

where $E_{m}^{t}$ is the residual error, $\delta \eta=0.5, k=20$.

By using Mathematical package BVPh2.0, we have reduced the average residual error. Different sets of global optimal convergence-control parameters are obtained at various approximations to achieve minimum values of related total averaged-squared residual error (see Table 1).

The average and total squared residual errors are defined in equations (30) to (33), respectively, and presented in Table 2 at diverse order of approximations. Increasing the order of approximations error can be reduced.

\section{Results and Discussion}

The variations of the profiles of the flow velocities, temperature, and concentration, as well as the skin-friction coefficients and the Nusselt and Sherwood numbers against the ratio of angular velocities $\gamma$, ratio of the buoyancy force $N, \varepsilon_{1}, \varepsilon_{2}$, and $\varepsilon_{3}$ are third-grade parameter, and the unsteadiness parameter $s$ are discussed through graphs and numerical tables.

Figures 2(a) to 2(d) describe the behavior of the tangential velocity $-f^{\prime}(\eta)$ for, $\varepsilon_{1}, \varepsilon_{2}$, and $\varepsilon_{3}$. It is found from Figure 2(a) that for $\gamma=0.5$ the fluid and the cone are rotating in the same direction with equal angular velocity and only because of favorable gradient pressure, i.e., $\lambda_{1}=1$. When $\gamma>0.5$, the velocity $-f^{\prime}(\eta)$ increases its magnitude while it decreases for $\gamma<0.5$. The velocity $-f^{\prime}(\eta)$ at the edge of the boundary layer can be seen asymptotically, when $\gamma<0$ is reached in oscillatory fashion. In the physical area of the boundary layer, such oscillations which arise from the excess convection of the angular momentum. Figures 2(b) and 2(c) elucidate that the magnitude of the velocity $-f^{\prime}(\eta)$ reduces for a second-grade parameter $\varepsilon_{1}$, while there is an increase in the magnitude for $\varepsilon_{2}$. The velocity $-f^{\prime}(\eta)$ is a decreasing function of $\varepsilon_{3}$ (see Figure 2(d)).

The influences of $\varepsilon_{1}, \varepsilon_{2}$, and $\varepsilon_{3}$ on the velocity $g(\eta)$ is presented in Figures 3(a) to 3(d). Figure 3(a) displays that the velocity $g(\eta)$ decreases for $\gamma>0.5$, where as it is reversed when $\gamma<0.5$. It is evident in Figures 3(b) and 3(c) that the velocity $g(\eta)$ enhances with an increase in $\varepsilon_{1}$ and $\varepsilon_{2}$, respectively. It is seen from Figure 3(d) that $\varepsilon_{3}$ causes a reduction in the magnitude of the velocity $g(\eta)$. There is a rising behavior in the Nusselt and Sherwood numbers for increasing $N$ and $s$ (see Figures 4(a) and 4(b)). 
TABLE 1: Convergence parameters and residual errors using BVPh2.0.

\begin{tabular}{lcccccc}
\hline$m$ & $c_{0}^{f}$ & $c_{0}^{g}$ & $c_{0}^{\theta}$ & $c_{0}^{\phi}$ & $E_{m}^{t}$ & CPU time (S) \\
\hline 2.0 & -1.21 & -0.39 & -0.78 & -0.82 & $8.01 \times 10^{-4}$ & 6.73 \\
4.0 & -1.15 & -0.31 & -0.73 & -0.75 & $2.24 \times 10^{-5}$ & 83.32 \\
6.0 & -1.03 & -0.22 & -0.68 & -0.71 & $4.03 \times 10^{-6}$ & 5120.28 \\
8.0 & -0.98 & -0.19 & -0.62 & -0.67 & $1.38 \times 10^{-7}$ & 8592.14 \\
\hline
\end{tabular}

TABle 2: Residual errors using optimal values at $m=8$ from Table 1.

\begin{tabular}{lccccc}
\hline$m$ & $E_{m}^{f}$ & $E_{m}^{g}$ & $E_{m}^{\theta}$ & $E_{m}^{\phi}$ & CPU time (S) \\
\hline 6.0 & $7.96 \times 10^{-5}$ & $3.40 \times 10^{-5}$ & $3.22 \times 10^{-6}$ & $4.74 \times 10^{-6}$ & 13.61 \\
12.0 & $3.12 \times 10^{-6}$ & $3.12 \times 10^{-7}$ & $4.89 \times 10^{-8}$ & $3.92 \times 10^{-8}$ & 122.47 \\
18.0 & $5.02 \times 10^{-8}$ & $1.78 \times 10^{-8}$ & $8.57 \times 10^{-9}$ & $7.09 \times 10^{-9}$ & 280.7 \\
\hline
\end{tabular}

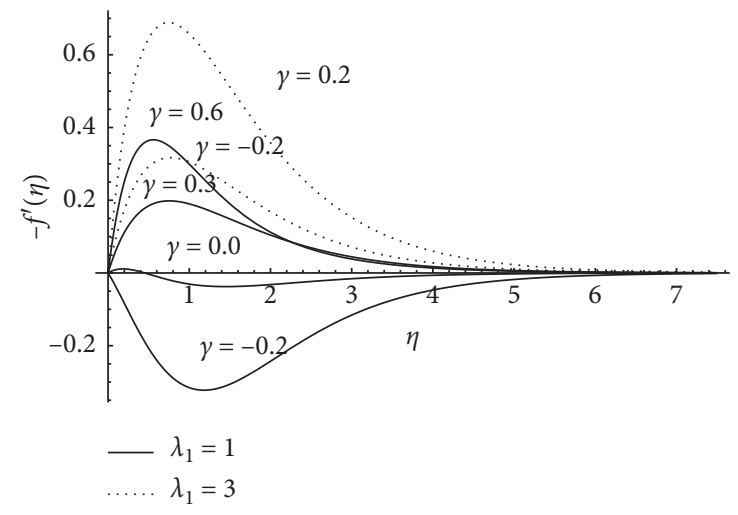

(a)

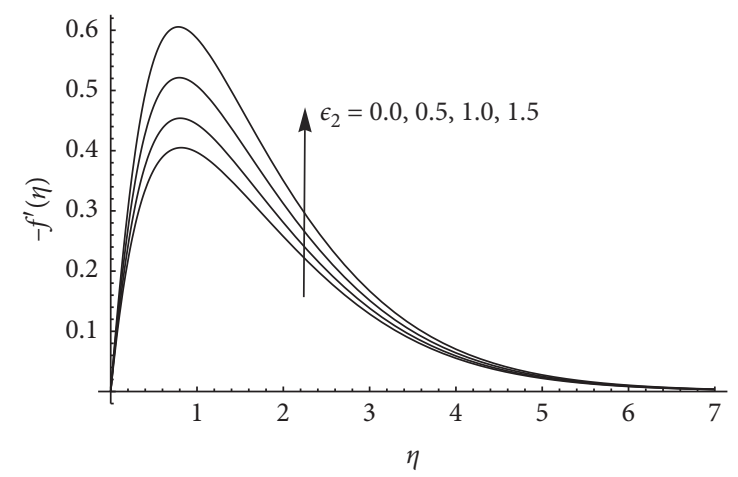

(c)

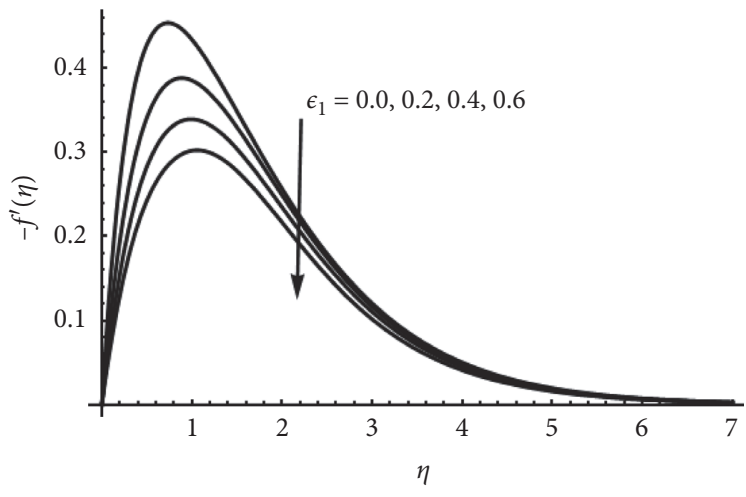

(b)

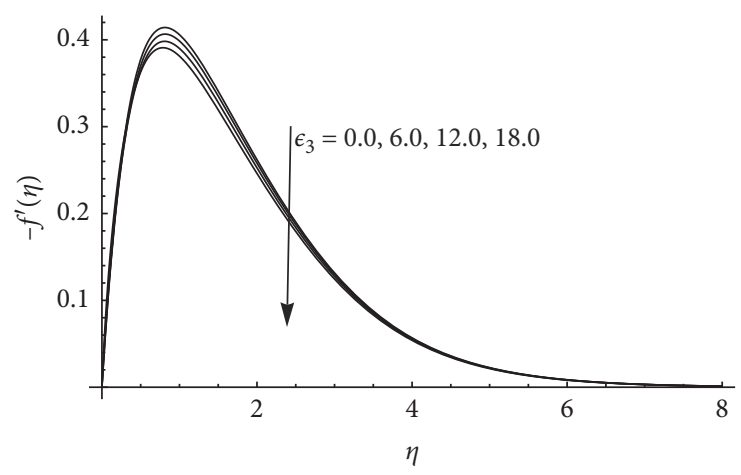

(d)

FIGURE 2: (a) Variation of $v$ for $\gamma$ and $\lambda_{1}$. (b) Variation of $v$ for $\varepsilon_{1}$. (c) Variation of tangential velocity $-f^{\prime}(\eta)$ for $\varepsilon_{2}$. (d) Variation of tangential velocity $-f^{\prime}(\eta)$ for $\varepsilon_{3}$.

Table 3 is reported in order to confirm the serious solutions obtained. Table 3 indicates that the analysis and numerical findings follow the appropriate criteria. The numerical values of the skin-friction coefficients in both directions for different emerging parameters are displayed in Table 4 . The influence of $\varepsilon_{1}, \varepsilon_{2}, \varepsilon_{3}$, and $s$ is to decrease the primary skin-friction coefficient. On the other hand, the secondary skin-friction coefficient varies directly with $\varepsilon_{1}, \varepsilon_{3}$, and $s$ but inversely proportional to $\varepsilon_{2}$. 


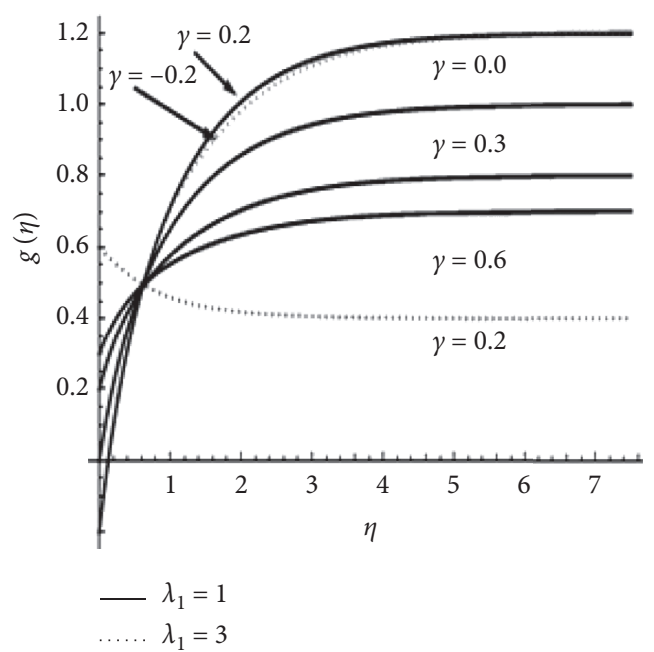

(a)

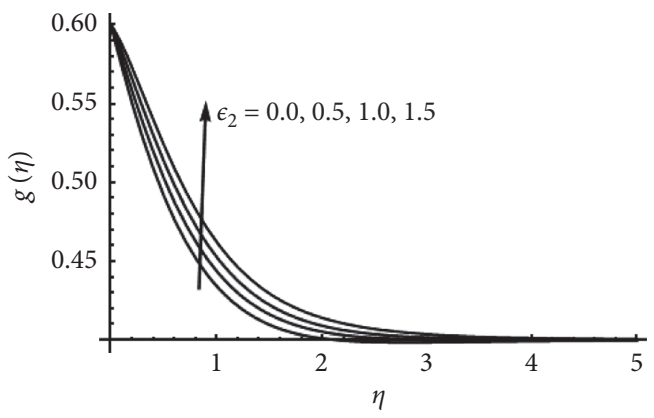

(c)

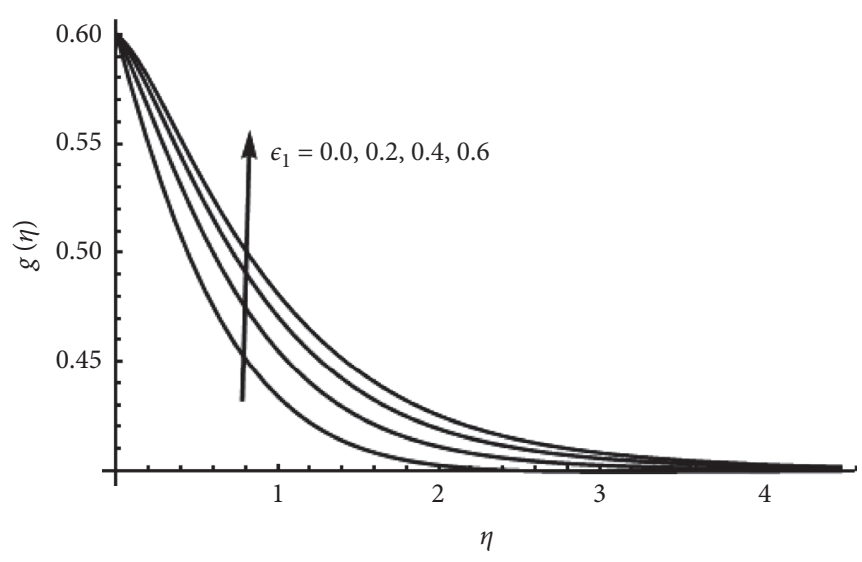

(b)

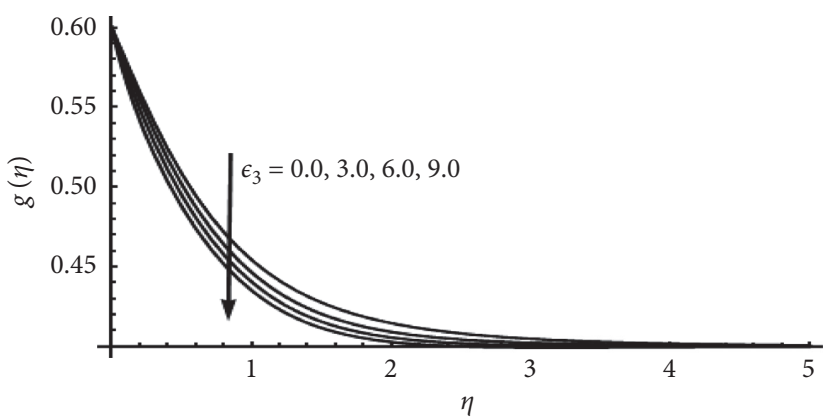

(d)

FIGURE 3: (a) Variation of azimuthal velocity $g(\eta)$ for $\gamma$ and $\lambda_{1}$. (b) Variation of azimuthal velocity $g(\eta)$ for $\varepsilon_{1}$. (c) Variation of azimuthal velocity $g(\eta)$ for $\varepsilon_{2}$. (d) Variation of azimuthal velocity $g(\eta)$ for $\varepsilon_{3}$.

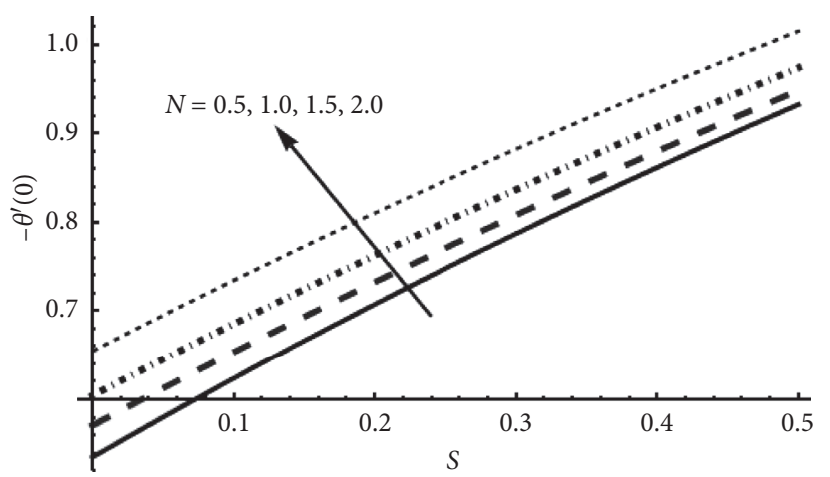

(a)

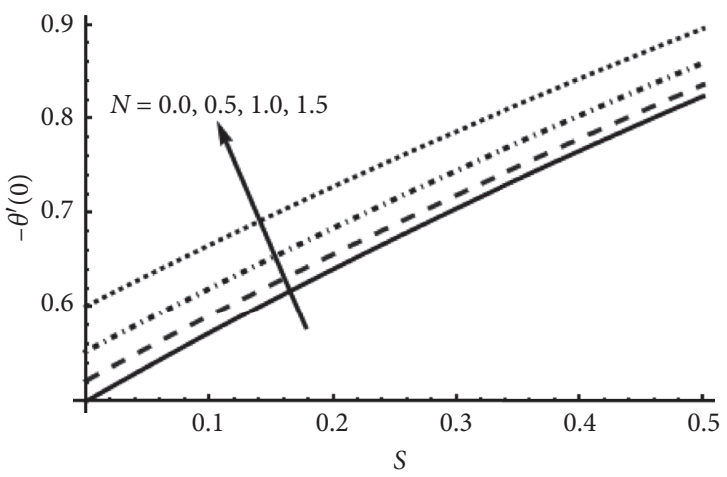

(b)

Figure 4: (a) Variation of $-\theta^{\prime}(0)$ for $N$. (b) Variation of $-\phi^{\prime}(0)$ for $N$.

TABle 3: Comparison of physical quantities for special case.

\begin{tabular}{|c|c|c|c|c|c|c|c|c|c|}
\hline \multirow[b]{2}{*}{$\operatorname{Pr}$} & \multirow[b]{2}{*}{ Sc } & \multicolumn{4}{|c|}{ Present results } & \multicolumn{4}{|c|}{ Numerical results [9] } \\
\hline & & $C_{f x} \operatorname{Re}_{x}^{1 / 2}$ & $C_{f y} \operatorname{Re}_{x}^{1 / 2}$ & $\mathrm{NuRe}_{x}^{-1 / 2}$ & $\mathrm{ShRe}_{x}^{-1 / 2}$ & $C_{f x} \operatorname{Re}_{x}^{1 / 2}$ & $C_{f y} \operatorname{Re}_{x}^{1 / 2}$ & $\mathrm{NuRe}_{x}^{-1 / 2}$ & $\operatorname{ShRe}_{x}^{-1 / 2}$ \\
\hline \multirow{3}{*}{0.7} & 0.22 & 1.55440 & -0.18623 & 0.92497 & 0.52523 & 1.55440 & -0.18623 & 0.92497 & 0.52523 \\
\hline & 0.60 & 1.39262 & -0.21530 & 0.90101 & 0.83546 & 1.39262 & -0.21530 & 0.90101 & 0.83546 \\
\hline & 0.94 & 1.31339 & -0.22754 & 0.86680 & 1.02810 & 1.31339 & -0.22754 & 0.86680 & 1.02810 \\
\hline \multirow{3}{*}{3.0} & 0.22 & 1.28598 & -0.21421 & 1.79674 & 0.50415 & 1.28590 & -0.21429 & 1.79674 & 0.50415 \\
\hline & 0.60 & 1.12925 & -0.24888 & 1.76655 & 0.80323 & 1.12920 & -0.24890 & 1.76657 & 0.80323 \\
\hline & 0.94 & 1.05234 & -0.26443 & 1.75182 & 0.99102 & 1.05237 & -0.26441 & 1.75183 & 0.99103 \\
\hline
\end{tabular}


TABLE 4: variation of surface skin-friction coefficients.

\begin{tabular}{|c|c|c|c|c|c|}
\hline$\varepsilon_{1}$ & $\varepsilon_{2}$ & $\varepsilon_{3}$ & $s$ & $C_{f x} \operatorname{Re}_{x}^{1 / 2}$ & $C_{f y} \operatorname{Re}_{x}^{1 / 2}$ \\
\hline 0.0 & & & & -0.00396 & 0.20191 \\
\hline 0.2 & & & & -0.05361 & 0.26225 \\
\hline 0.6 & & & & -0.10326 & 0.38293 \\
\hline & 0.0 & & & -0.00242 & 0.23220 \\
\hline & 1.0 & & & -0.06985 & 0.23101 \\
\hline & 2.0 & & & -0.11548 & 0.22982 \\
\hline & & 0.0 & & -0.02825 & 0.22988 \\
\hline & & 0.5 & & -0.03083 & 0.25381 \\
\hline & & 1.5 & & -0.03228 & 0.26734 \\
\hline & & & -0.5 & -0.02790 & 0.18665 \\
\hline & & & 0.0 & -0.02818 & 0.20180 \\
\hline & & & 0.5 & -0.02849 & 0.21694 \\
\hline
\end{tabular}

\section{Conclusions}

The problem of a third-grade fluid's combined convection flow is analyzed in the current work on a vertical rotating cone. The reduced nondimensional differential equations of third-grade fluid, temperature, and concentration are unraveled by the optimal homotopy analysis method (OHAM). The existing calculated outcomes are acknowledged in a conventional agreement with the previously published results available in the literature:

(i) It is found that the tangential velocity has a reducing manner for $\varepsilon_{1}$ and $\varepsilon_{3}$, however increases for $\varepsilon_{2}$

(ii) The skin-friction coefficients are increasing functions of the ratio of the buoyancy forces $N$

(iii) The impact of third-grade fluid parameters $\varepsilon_{1}, \varepsilon_{2}, \varepsilon_{3}$, and $s$ is found to decrease the primary skin-friction coefficient

(iv) There is increasing activity with Nusselt and Sherwood numbers for increasing $N$ values

\section{Data Availability}

No data were used to support this study.

\section{Conflicts of Interest}

The author declares that they have no conflicts of interest.

\section{Acknowledgments}

The author extends his appreciation to the Deanship of Scientific Research at King Khalid University for funding this work through research groups program under grant no. RGP.2/38/42.

\section{References}

[1] U. Nazir, S. Saleem, M. Nawaz, M. A. Sadiq, and A. A. Alderremy, "Study of transport phenomenon in Carreau fluid using Cattaneo-Christov heat flux model with temperature dependent diffusion coefficients," Physica A: Statistical Mechanics and its Applications, vol. 554, p. 123921, 2020.

[2] O. D. Makinde and T. Chinyoka, "Numerical study of unsteady hydromagnetic generalized Couette flow of a reactive third-grade fluid with asymmetric convective cooling," Computers \& Mathematics with Applications, vol. 61, no. 4, pp. 1167-1179, 2011.

[3] R. Ellahi, T. Hayat, and F. M. Mahomed, "Generalized Couette flow of a third-grade fluid with slip: the exact solutions," Zeitschrift für Naturforschung A, vol. 65, no. 12, pp. 1071-1076, 2010.

[4] T. Salahuddin, M. Y. Malik, A. Hussain, S. Bilal, and M. Awais, "MHD flow of Cattanneo-Christov heat flux model for Williamson fluid over a stretching sheet with variable thickness: using numerical approach," Journal of Magnetism and Magnetic Materials, vol. 401, pp. 991-997, 2016.

[5] A. Attia, A. L. Aboul-Hassan, M. A. M. Abdeen, A. El-Din Abdin, and W. Abd El-Meged, "Unsteady Couette flow of a thermally conducting viscoelastic fluid under constant pressure gradient in a porous medium," Chinese Journal of Physics, vol. 52, pp. 1015-1027, 2014.

[6] P. M. Krishna, N. Sandeep, J. V. R. Reddy, and V. Sugunamma, "Dual solutions for unsteady flow of PowellEyring fluid past an inclined stretching sheet," Journal of Naval Architecture and Marine Engineering, vol. 13, no. 1, pp. 89-99, 2016.

[7] T. Hayat, M. Waqas, M. I. Khan, and A. Alsaedi, "Impacts of constructive and destructive chemical reactions in magnetohydrodynamic (MHD) flow of Jeffrey liquid due to nonlinear radially stretched surface," Journal of Molecular Liquids, vol. 225, pp. 302-310, 2017.

[8] R. Ellahi, M. M. Bhatti, and I. Pop, "Effects of hall and ion slip on MHD peristaltic flow of Jeffrey fluid in a non-uniform rectangular duct," International Journal of Numerical Methods for Heat \& Fluid Flow, vol. 26, no. 6, pp. 1802-1820, 2016.

[9] D. Anilkumar and S. Roy, "Unsteady mixed convection flow on a rotating cone in a rotating fluid," Applied Mathematics and Computation, vol. 155, no. 2, pp. 545-561, 2004.

[10] S. Saleem, H. Firdous, S. Nadeem, and A. U. Khan, "Convective heat and mass transfer in magneto Walter's B nanofluid flow induced by a rotating cone," Arabian Journal for Science and Engineering, vol. 44, no. 2, pp. 1515-1523, 2019.

[11] S. A. Khan, T. Hayat, M. Ijaz Khan, and A. Alsaedi, "Salient features of Dufour and Soret effect in radiative MHD flow of viscous fluid by a rotating cone with entropy generation," International Journal of Hydrogen Energy, vol. 45, no. 28, pp. 14552-14564, 2020.

[12] M. A. Hossain and S. C. Paul, "Free convection from a vertical permeable circular cone with non-uniform surface temperature," Acta Mechanica, vol. 151, no. 1-2, pp. 103-114, 2001.

[13] A. J. Chamkha and A. Al-Mudhaf, "Unsteady heat and mass transfer from a rotating vertical cone with a magnetic field and heat generation or absorption effects," International Journal of Thermal Sciences, vol. 44, no. 3, pp. 267-276, 2005.

[14] S. Nadeem and S. Saleem, "Series solution of unsteady Eyring Powell nanofluid flow on a rotating cone," Indian Journal of Pure and Applied Physics, vol. 52, pp. 725-737, 2014.

[15] F. T. Zohraa, M. J. Uddin, A. I. M. Ismail, O. A. Beg, and A. Kadir, "Anisotropic slip magneto-bioconvection flow from a rotating cone to a nanofluid with Stefan blowing effects," Chinese Journal of Physics, vol. 56, p. 432e48, 2018.

[16] S. Saleem, S. Nadeem, and R. Ul Haq, "Buoyancy and metallic particle effects on an unsteady water-based fluidflow along a vertically rotating cone," The European Physical Journal Plus, vol. 129, p. 213, 2014.

[17] C. S. K. Raju and N. Sandeep, "Unsteady Casson nanofluid flow over a rotating cone in a rotating frame filled with ferrous 
nanoparticles: a numerical study," Journal of Magnetism and Magnetic Materials, vol. 421, p. 216e24, 2017.

[18] A. J. Chamkha and A. M. Rashad, "Unsteady heat and mass transfer by MHD mixed convection flow from a rotating vertical cone with chemical reaction and Soret and Dufour effects," The Canadian Journal of Chemical Engineering, vol. 92, no. 4, pp. 758-767, 2014.

[19] S. Liao, "An optimal Homotopy-analysis approach for strongly nonlinear differential equations," Communications in Nonlinear Science and Numerical Simulation, vol. 15, no. 8, pp. 2003-2016, 2010.

[20] S. Nadeem and S. Saleem, "Analytical treatment of unsteady mixed convection MHD flow on a rotating cone in a rotating frame," Journal of the Taiwan Institute of Chemical Engineers, vol. 44, no. 4, pp. 596-604, 2013.

[21] M. Waqas, M. Ijaz Khan, T. Hayat, A. Alsaedi, and M. Imran Khan, "On Cattaneo-Christov double diffusion impact for temperature-dependent conductivity of Powell-Eyring liquid," Chinese Journal of Physics, vol. 55, no. 3, p. 729, 2017.

[22] M. M. Rashidi, N. Vishnu Ganesh, A. K. Abdul Hakeem, and B. Ganga, "Buoyancy effect on MHD flow of nanofluid over a stretching sheet in the presence of thermal radiation," Journal of Molecular Liquids, vol. 198, pp. 234-238, 2014.

[23] P. Agarwal, M. Akbar, R. Nawaz, and M. Jleli, "Solutions of system of Volterra integro-differential equations using optimal homotopy asymptotic method," Mathematical Methods in the Applied Sciences, vol. 44, no. 3, pp. 2671-2681, 2020.

[24] A. Zeeshan, A. Majeed, and R. Ellahi, "Effect of magnetic dipole on viscous ferro-fluid past a stretching surface with thermal radiation," Journal of Molecular Liquids, vol. 215, pp. 549-554, 2016.

[25] K. M. Saad, O. S. Iyiola, and P. Agarwal, "An effective homotopy analysis method to solve the cubic isothermal autocatalytic chemical system," AIMS Mathematics, vol. 3, no. 1, pp. 183-194, 2018.

[26] Z. Odibat, "An improved optimal homotopy analysis algorithm for nonlinear differential equations," Journal of Mathematical Analysis and Applications, vol. 488, no. 2, p. 124089, 2020.

[27] R. Mehmood, S. Nadeem, S. Saleem, and N. S. Akbar, "Flow and heat transfer analysis of Jeffery nano fluid impinging obliquely over a stretched plate," Journal of the Taiwan Institute of Chemical Engineers, vol. 74, pp. 49-58, 2017. 